Pacific Journal of Mathematic 


\section{GENERATORS OF FACTORS OF BERNOULLI SHIFTS}

\section{LAIF SWANSON}

One of the questions of ergodic theory is that of "relative position" of factors of Bernoulli shift. If $\mathscr{F}_{0}$ and $\mathscr{F}_{1}$ are factor algebras for a Bernoulli shift $T$, under what conditions is there an isomorphism $\phi$ commuting with $T$ such that $\phi \mathscr{T}_{0}=\mathscr{T}_{1}$ ?

In this paper, we give an example of a Bernoulli shift $T$ of a space $X$ and uncountably many partitions $\left\{Q_{\alpha}: \alpha \in A\right\}$ of $X$ with the properties:

(1) $\left(T, Q_{\alpha}\right\} \cong\left(T, Q_{\beta}\right)$ for $\alpha, \beta \in A$.

(2) $\bigvee_{-\infty}^{\infty} T^{i} Q_{\alpha}$ is maximal for its entropy whenever $\alpha \in A$.

(3) There is no isomorphism $\phi$ commuting with $T$ such that $\phi Q_{\alpha}=Q_{\beta}$ unless $\alpha=\beta$.

If $T$ is an automorphism of a probability space $(X, \mathscr{F}, \mu)$, a sub-sigma algebra $\mathscr{F}_{0}$ of $\mathscr{F}$ is a factor algebra for $T$ if $T$ is an automorphism of $\left(X, \mathscr{F}_{0}, \mu\right)$. That is, $\mathscr{F}_{0}$ is a factor algebra for $T$ means $A \in \mathscr{F}_{0} \Leftrightarrow T A \in \mathscr{F}_{0}$. If $\mathscr{F}_{0}$ is a factor algebra for $T$, the automorphism $T$ restricted to $\left(X, \mathscr{F}_{0}, \mu\right)$ is called a factor of $T$, denoted $T \mid \mathscr{F}_{0}$.

It is clear that factors of ergodic, weakly mixing, mixing, or Kolmogorov automorphisms are ergodic weakly mixing, mixing, or Kolmogorov respectively. It is known (Ornstein) that factors of Bernoulli shifts are Bernoulli. We investigate the "relative position" of factors of Bernoulli shifts.

This paper is part of a $\mathrm{Ph}$. $\mathrm{D}$. thesis prepared under the supervision of Jacob Feldman. I thank him for many helpful discussions as well as his encouragement. Thanks are also due Donald Ornstein who suggested this problem and listened to my ideas.

Some of the questions one might ask about factors of Bernoulli shifts are:

(1) If $T$ and $T^{\prime}$ are isomorphic Bernoulli shifts (on spaces $X$ and $X^{\prime}$ ) with respective factor algebras $\mathscr{F}_{0}$ and $\mathscr{F}_{0}^{\prime}$, under what conditions is there an isomorphism $\phi$ (defined except on a set of measure zero) such that

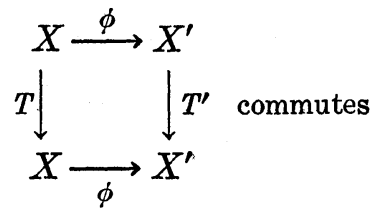


(i.e., $\phi$ is an isomorphism of $T$ and $T^{\prime}$ ) and

$$
\phi\left(\mathscr{F}_{0}\right)=\mathscr{F}_{0}^{\prime}
$$

(i.e., $\left.A \in \mathscr{F}_{0} \Leftrightarrow \phi A \in \mathscr{F}_{0}\right)$ ?

(2) If $T$ and $T^{\prime}$ are Bernoulli shifts (on spaces $X$ and $X^{\prime}$ respectively), $Q$ is a partition of $X$ and $Q^{\prime}$ a partition of $X^{\prime}$, under what conditions is there an isomorphism $\phi$ of $T$ and $T^{\prime}$ so that $\phi Q=Q^{\prime}$, (i.e. $Q=\left(Q_{1}, \cdots, Q_{n}\right), Q^{\prime}=\left(Q_{i}, \cdots, Q_{n}^{\prime}\right)$, and $\phi Q_{i}=Q_{i}^{\prime}$ for each $i)$ ?

(3) If $T$ is a Bernoulli shift and $\mathscr{F}_{0}$ a factor algebra for $T$, under what conditions is there another factor algebra $\mathscr{F}_{1}$ so that

$$
\mathscr{F}_{0} \perp \mathscr{F}_{1}
$$

and

$$
\mathscr{F}_{0} V \mathscr{F}_{1}=\mathscr{F} ?
$$

(We will call such a factor algebra $\mathscr{F}_{1}$ a complement of $\mathscr{F}_{0}$ )

We can see a close relationship between questions (1) and (3): If we are given a Bernoulli shift $T$ of $X$ and a factor algebra $\mathscr{F}_{0}$ such that $h(T)>h\left(T \mid \mathscr{F}_{0}\right)$, denote by $T_{B}$ a Bernoulli shift of a space $Y$ such that $h\left(T_{B}\right)=h(T)-h\left(T \mid \mathscr{F}_{0}\right)$. Define $T^{\prime}=T \mid \mathscr{F}_{0} \times T_{B}$, and denote by $\mathscr{F}_{0}^{\prime}$ and $\mathscr{F}_{1}^{\prime}$, respectively, the $\sigma$-algebras of the first and second members of the product. Now, the Ornstein isomorphism theorem gives us the fact that $T$ and $T^{\prime}$ are isomorphic, and an isomorphism $\phi$ of $T$ and $T^{\prime}$ taking $\mathscr{F}_{0}$ to $\mathscr{F}_{0}^{\prime}$ would take some complement of $\mathscr{F}_{0}$ to $\mathscr{F}_{0}^{\prime}$, i.e., if $\phi$ is an isomorphism of $T$ and $T^{\prime}$ and $\phi\left(\mathscr{F}_{0}\right)=\mathscr{F}_{0}{ }^{\prime}$, then $\phi^{-1}\left(\mathscr{F}_{1}^{\prime}\right)$ is a complement for $\mathscr{F}_{0}$.

On the other hand, if $T \cong T^{\prime}$, and $\mathscr{F}_{0}$ and $\mathscr{F}_{0}^{\prime}$ are respective factor algebras satisfying

$$
\begin{aligned}
& h\left(T \mid \mathscr{F}_{0}\right)=h\left(T \mid \mathscr{F}_{0}^{\prime}\right) \\
& \mathscr{F}_{0} \text { is complemented by } \mathscr{F}_{1} \\
& \mathscr{F}_{0}^{\prime} \text { is complemented by } \mathscr{F}_{0}^{\prime}
\end{aligned}
$$

then the Ornstein isomorphism theorem gives isomorphisms

$$
\phi_{0} \text { from } T \mid \mathscr{F}_{0} \text { to } T^{\prime} \mid \mathscr{F}_{0}^{\prime}
$$

and

$$
\phi_{1} \text { from } T \mid \mathscr{F}_{1} \text { to } T^{\prime} \mid \mathscr{F}_{1}^{\prime} \text {; }
$$

and $\phi=\phi_{0} \times \phi_{1}$ is an isomorphism of $T$ and $T^{\prime}$ carrying $\mathscr{F}_{0}$ to $\mathscr{F}_{0}^{\prime}$. Thus questions (1) and (3) are related in this manner: If $\mathscr{F}_{0}$ and $\mathscr{F}_{0}^{\prime}$ are both complemented, there is an isomorphism from $T$ to $T^{\prime}$ 
taking $\mathscr{F}_{0}$ to $\mathscr{F}_{0}^{\prime}$. If either is complemented and such an isomorphism exists, they are both complemented.

We can also see that (2) is in a natural way a stronger question than (1): If $T$ and $T^{\prime}$ are isomorphic Bernoulli shifts, $\bigvee_{-\infty}^{\infty} T^{i} Q=\mathscr{F}_{0}$ and $V_{-\infty}^{\infty} T^{\prime} Q^{\prime}=\mathscr{F}_{0}^{\prime}$, then an isomorphism of $T$ and $T^{\prime}$ taking $Q$ to $Q^{\prime}$ will certainly take $\mathscr{F}_{0}$ to $\mathscr{F}_{0}^{\prime}$.

Some results about these questions are known. Thouvenot [7] has discovered conditions similar to the condition "finitely determined" (see Ornstein, [4], for this definition) which are equivalent to a factor of a Bernoulli shift being complemented, and has shown that for every Bernoulli shift $T$ and factor algebra $\mathscr{F}_{0}$, there is a factor algebra $\mathscr{F}_{1}$ satisfying the weaker conditions

$$
\begin{gathered}
\mathscr{F}_{0} \perp \mathscr{F}_{1} \\
h\left(T \mid \mathscr{F}_{0} V \mathscr{F}_{1}\right)=h(T) .
\end{gathered}
$$

On the other hand, Ornstein and Weiss [6] have shown that if $T$ is a Bernoulli shift, (in fact, any $K$-automorphism) and $\mathscr{F}_{0}, \mathscr{F}_{1}$, and $\mathscr{F}_{2}$ factor algebras for $T$ satisfying

$$
\begin{gathered}
\mathscr{F}_{0} \subset \mathscr{F}_{1} \\
h\left(T \perp \mathscr{F}_{0}\right)=h\left(T \perp \mathscr{F}_{1}\right)
\end{gathered}
$$

and

$$
\mathscr{F}_{0} \perp \mathscr{F}_{2}
$$

then

$$
\mathscr{F}_{1} \perp \mathscr{F}_{2} \text {. }
$$

Thus a factor algebra $\mathscr{F}_{0}$ for a Bernoulli shift $T$ can be complemented only if

$$
\mathscr{F}_{1} \supsetneq \mathscr{F}_{0} \Longrightarrow\left[h\left(T \mid \mathscr{F}_{1}\right)>h\left(T \mid \mathscr{F}_{0}\right)\right] \text {. }
$$

a condition which we will denote by " $\mathscr{F}_{0}$ is maximal for its entropy" since it means that if we expand $\mathscr{F}_{0}$ we increase the entropy of the factor.

So an immediate question might be "Is it true that if $T$ is a Bernoulli shift and $\mathscr{F}_{0}$ a factor algebra which is maximal with respect to its entropy, then $\mathscr{F}_{0}$ if complemented?" This is, as we noted earlier, the same question as "If $T$ and $T$ " are isomorphic Bernoulli shifts and $\mathscr{F}_{0}$ and $\mathscr{F}_{0}^{\prime}$ are respective factor algebras, each maximal 
with respect to its entropy, with $h\left(T \mid \mathscr{F}_{0}\right)=h\left(T^{\prime} \mid \mathscr{F}_{0}^{\prime}\right)$, is there an isomorphism $\phi$ of $T$ and $T^{\prime}$ so that $\phi\left(\mathscr{F}_{0}\right)=\mathscr{F}_{0}^{\prime}$ ?" The answer to these questions is no-a counterexample has been given by Ornstein [3]. This gives partial answers to questions one and two.

Since Ornstein's methods involve a skew product with a member of the uncountable family of non-Bernoulli $K$-automorphisms (OrnsteinShields, [5]), an immediate question is "Is there a Bernoulli shift with an uncountable number if factor algebras $\left\{\mathscr{F}_{\alpha}: \alpha \in A\right\}$ with the properties:

(i ) $\mathscr{F}_{\alpha}$ is maximal with respect to its entropy, for every $\alpha \in A$

(ii) $h\left(T \mid \mathscr{F}_{\alpha}\right)=h\left(T \mid \mathscr{F}_{\beta}\right)$ for every $\alpha, \beta \in A$

(iii) If $\alpha, \beta \in A$, there is no isomorphism $\phi$ commuting with $T$ such that $\phi\left(\mathscr{F}_{\alpha}\right)=\mathscr{F}_{\beta}$ unless $\alpha=\beta$ ?

We deal with a weaker question, like question (2): Is there an uncountable family $\left\{T_{\alpha}: \alpha \in A\right\}$ of isomorphic Bernoulli shifts of spaces $X_{\alpha}$ and partitions $Q_{\alpha}$ of $X_{\alpha}$ such that $\alpha \in A$

(i ) $\bigvee_{-\infty}^{\infty} T_{\alpha}^{i} Q_{\alpha}$ is maximal with respect to its entropy for every

(ii) $\left(T_{\alpha}, Q_{\alpha}\right) \cong\left(T_{\beta}, Q_{\beta}\right)$ for every $\alpha, \beta \in A$

(iii) If $\alpha, \beta \in A$, there is no isomorphism $\phi$ of $T_{\alpha}$ and $T_{\beta}$ such that $\phi\left(Q_{\alpha}\right)=Q_{\beta}$ unless $\alpha=\beta$ ?

The family of processes constructed by Ornstein [3] answers this question. Each automorphism is the skew product of a certain Bernoulli shift with one of the non-Bernoulli $K$-automorphisms and the identity. These automorphisms are proved Bernoulli by Ornstein [3]. We assume a familiarity with the uncountable family of $K$ automorphisms.

The construction. We first construct a Bernoulli shift $T_{B}$; we will then skew this with a non-Bernoulli $K$-automorphism and the identity.

$T_{B}$ is the common extension of the gadget transformations for a sequence $G(n)$ of gadgets which are defined inductively. It is generated by a partition $R=\left(R_{0}, R_{1}, R_{e}, R_{f}\right)$ which also denotes the partition associated with each gadget. The gadgets depend on certain increasing sequences of integers $f(n), k(n)$, and $s(n) . G(1)$ is $\left(A_{1}, \cdots, A_{p}\right)$ and $G^{*}(1) \subset R_{0}$.

We will describe $T_{B}$ inductively by assuming that $G(n)$ has already been constructed and constructing $G(n+1)$ :

Divide $G(n)$ into $s(n)$ isomorphic gadgets $G(n, 1), \cdots, G(n, s(n))$. 
Let $\bar{G}(n+1)=G(n, 1) * \cdots * G(n, s(n))$.

Divide each column $C$ of $\bar{G}(n+1)$ into $(f(n)-1) k(n)$ columns $C(i, j), 1 \leqq i \leqq f(n), 1 \leqq j \leqq k(n)$. Precede $C(i, j)$ by $j$ intervals of $R_{0}, k(n)-j$ intervals of $R_{1}$, and $i$ intervals $R_{f}$, and follow it by $f(n)-i$ intervals of $R_{e}, k(n)-j$ intervals of $R_{0}$, and $j$ intervals of $R_{1}$. These columns are the columns of $G(n+1)$.

So a typical column of $G(n)$ looks like:

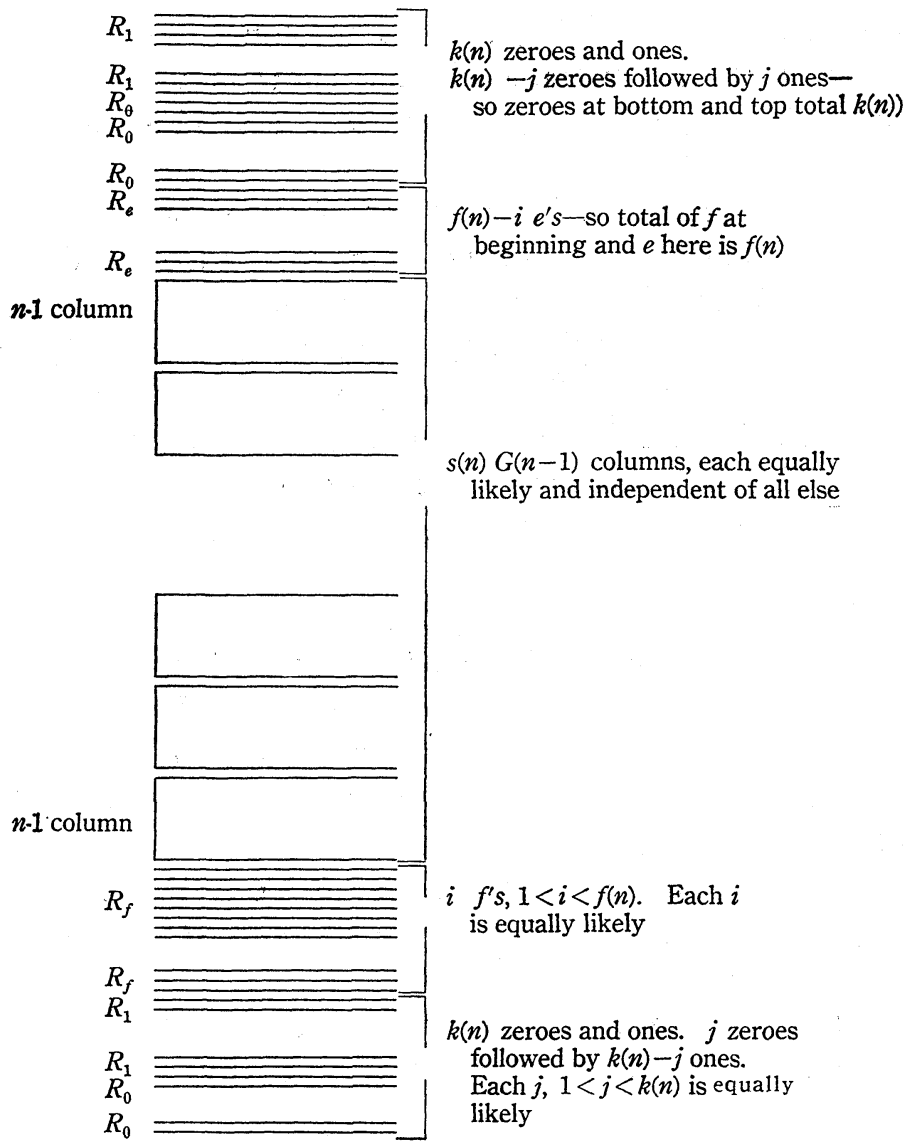

We use $X$ to denote $\bigcup_{n} G^{*}(n)$, and so $T_{B}$ is an automorphism of $X$. Let $\bar{T}_{\alpha}$ be any of the non-Bernoulli $K$-automorphisms defined by Ornstein and Shields, and let $Y_{\alpha}$ be the space on which $\bar{T}_{\alpha}$ is defined. We define an automorphism, which we call $T_{\alpha}$, of the space $X \times Y_{\alpha}$ (with the product $\sigma$-algebra and measure) by

$$
T_{\alpha}(x, y)=\left\{\begin{array}{lll}
\left.T_{B} x, \bar{T}_{\alpha} y\right) & \text { if } \quad x \in R_{0} \\
\left.T_{B} x, y\right) & \text { if } \quad x \notin R_{0}
\end{array}\right.
$$


Ornstein shows that this automorphism is a Bernoulli shift of $X \times Y_{\alpha}$ if sequences $f, s$, and $k$ satisfy certain conditions which will cause, among other things, the number of zeroes in an $n$-block for $T_{B}$ to be large compared to the length of an $n$-block for $T_{\alpha}$ and small compared to the length of an $(n+1)$-block for $T_{\alpha}$.

We define a partition $Q^{\alpha}=\left(Q_{0}^{\alpha}, Q_{1}^{\alpha}, Q_{e}^{\alpha}, Q_{f}^{\alpha}\right)$ of $X \times Y_{\alpha}$ by

$$
\begin{aligned}
& Q_{0}^{\alpha}=\left\{(x, y) \in X \times Y_{\alpha}: x \in R_{0}\right\} ; Q_{1}^{\alpha}=\left\{(x, y) \in X \times Y_{\alpha}: x \in R_{1}\right\} \\
& Q_{e}^{\alpha}=\left\{(x, y) \in X \times Y_{\alpha}: x \in R_{e}\right\} ; Q_{f}^{\alpha}=\left\{(x, y) \in X \times Y_{\alpha}: x \in R_{f}\right\} .
\end{aligned}
$$

Ornstein shows that $\bigvee_{-\infty}^{\infty} T^{i} Q^{\alpha}$ is maximal with respect to its entropy, and is not complemented, i.e., there is no factor algebra $\mathscr{F}_{1}$ for $T_{\alpha}$ so that $V_{-\infty}^{\infty} T_{\alpha}^{i} Q^{\alpha} \perp \mathscr{F}_{1}$ and $V_{-\infty}^{\infty} T_{\alpha}^{i} Q^{\alpha} V \mathscr{F}_{1}$ is the product $\sigma$-algebra for $X \times Y_{\alpha}$.

We will show that if a "family" of $K$-automorphisms, depending on sequences $f$ and $s$ of integers, is fixed, and $\bar{T}_{\alpha}$ and $\bar{T}_{\beta}$ are nonisomorphic members of this family, then there is no isomorphism $\phi$ of $\bar{T}_{\alpha}$ and $\bar{T}_{\beta}$ such that $\phi\left(Q^{\alpha}\right)=Q^{\beta}$. (Note that since $\bar{T}_{\alpha}$ and $\bar{T}_{\beta}$ are from the same family, $h\left(\bar{T}_{\alpha}\right)=h\left(\bar{T}_{\beta}\right)$, and so $h\left(T_{\alpha}\right)=h\left(T_{\beta}\right)$, and so, by the Ornstein isomorphism theorem, $T_{\alpha} \cong T_{\beta}$.) We will show the nonexistence of $\phi$ by assuming that there is such a $\phi$ and proving that $\bar{T}_{\alpha} \cong \bar{T}_{\beta}$, using the following facts about the family of $K$ automorphisms.

(1) $\bar{T}_{\alpha}=\bar{T}_{\beta}$ if and only if $g_{\alpha}(n)$ for all but finitely many $n$.

(2) Let $R^{\beta, k}$ be the partition of $Y_{\beta}$ defined by $R^{\beta, k}=\left(R_{0}^{\beta, k}, R_{1}^{\beta, k}\right)$ where $R_{1}^{\beta, k}=G^{*}(k)$. There is a certain number $\bar{\varepsilon}$ with the property that if $R^{k}$ is any partition of $Y_{\alpha}, y_{\alpha} \in Y_{\alpha}$ and $y_{\beta} \in Y_{\beta}$ and the $R^{k}$ name of $y_{\alpha}$ agrees with the $R^{\beta, k}$-name of $y_{\beta}$ in all but $\bar{\varepsilon}$ places, then $g_{\alpha}(k)=g_{\beta}(k)$.

Let $p_{1}$ and $p_{2}$ be the projections of $X \times Y_{\alpha}$ onto $X$ and $Y_{\alpha}$ respectively. Since $T_{\beta} \phi=\phi T_{\alpha}, \phi Q^{\alpha}=Q^{\beta}$, and $V_{-\infty}^{\infty} T_{\alpha}^{i} Q^{\alpha}=\phi\left(p_{1}\right)$, we see that $p_{1}(\phi(x, y))=x$.

Recall that $Y_{\alpha}$ is partitioned by $P=\left(P_{e}, P_{f}, P_{s}\right)$ which generates for $\bar{T}_{\alpha}$. We define a partition $P^{\alpha}$ of $X \times Y_{\alpha}$ by

$$
\begin{aligned}
& (x, y) \in P_{e}^{\alpha} \Longleftrightarrow y \in P_{e} \\
& (x, y) \in P_{f}^{\alpha} \Longleftrightarrow y \in P_{f} \\
& (x, y) \in P_{s}^{\alpha} \Longleftrightarrow y \in P_{s} .
\end{aligned}
$$

Let $P^{\prime}$ be the partition $\phi^{-1}\left(P^{\beta}\right)$ of $X \times Y_{\alpha}$. We can make new "names" $\bar{P}$ and $\bar{P}^{\prime}$ for the points of $X \times Y_{\alpha}$ by looking at the $P$ and $P^{\prime}$ names, respectively, in only those positions for which the $Q^{\alpha}$ name is $Q_{0}^{\alpha}$. Of course these "names" do not properly shift with $T_{\alpha}$. The $\bar{P}_{\alpha}$ name of $(x, y)$ is the $P$ name of $y$; the $\bar{P}^{\prime}$ name of $(x, y)$ is the $P$ name of $P_{2}(\phi(x, y))$. 
We know, since $Q^{\alpha} V P^{\alpha}$ is a generator for $T_{\alpha}$, that $P^{\prime}$ names can be coded from $Q^{\alpha} V P^{\alpha}$ names. That is, for every $\varepsilon>0$ there is an $n=n(\varepsilon)$ and a partition $P_{1} \subset \mathrm{V}_{-n}^{n} T_{\alpha}\left(Q^{\alpha} V P^{\alpha}\right)$ such that $\left|P_{1}-P^{\prime}\right|<\varepsilon$.

Let $R^{k, x}$ be the partition $\left(R_{0}^{k, x}, R_{1}^{k, x}\right)$ of $Y_{\alpha}$ where

$$
R_{1}^{k, x}=\left\{y_{\alpha} \in Y_{\alpha}: p_{2} \phi\left(x, y_{\alpha}\right) \in G^{*}(k)\right\} \text {. }
$$

Let $k$ be large enough that the length of a $(k-1)$ block for $T_{B}$ is at least twice $n(\bar{\varepsilon} / 4)$; that is, choose $k$ so large that strings in the $Q^{\alpha} V P^{\alpha}$ name which are as long as a $(k-1)$ block for $T_{B}$ predict $P^{\prime}$ with accuracy at least $1-(\bar{\varepsilon} / 4)$. We will show that whether these strings predict $R_{1}^{k, x} R_{0}^{k, x}$ is nearly independent of $x$.

Let $A$ be a $Q^{\alpha} V P^{\alpha}$ string whose $Q^{\alpha}$ part is two consecutive $k$ blocks and whose $P$ part and $P^{\prime}$ part are mostly $k$-blocks in the same $(k+1)$-block. (Most strings of this length have their $\bar{P}$-part and $\bar{P}^{\prime}$ part made up mostly of $k$-blocks in the same $(k+1)$-block.) So we have chosen $A$ so that it is the set of points $(x, y)$ which for a certain $j$ have $T_{B}^{j} x$ in the base of a particular column of $\mathscr{F}(k)$, and have $T_{B}^{j+h(k)} x$ in the base of a particular column of $\mathscr{F}(k)$, and whose $P_{\alpha}$ and $P^{\prime}$ names in the positions $j$ through $j+2 h(k)$ are made mostly of certain $k$-blocks in the same $(k+1)$-block.

Let $B$ be another $Q^{\alpha} V P^{\alpha}$ string whose $\bar{P}^{\alpha}$ part is the same as for $A$ and whose $Q^{\alpha}$ part is the same as $A^{\prime}$ 's except that the number of zeroes at the beginning of the second $k$-block is larger. That is, $B$ is the set of $(x, y)$ for which $T_{B}^{j} x$ is in the base of the same column for $\mathscr{F}(k)$ as for $A$, but $T_{B}^{j+h(k)} x$ is in a column which begins with $z$ more zeroes than the corresponding column for $A$. Thus, the $P^{\alpha}$ part of $B$ is "bunched up" at the beginning of the second $k$-block when you compare it to that of $A$.

Since the first halves of the strings $A$ and $B$ are the same, the positions for $k$-blocks in the $\bar{P}^{\prime}$ name of $B$ must be the same as for $A$ (because of the rigidity of the block structure for $\bar{T}_{B}$ ). Thus the "predictions" of $R^{k, x}$ and $R^{k, x^{\prime}}$, where $x^{\prime}=T_{B}^{Z} x$, based on strings of length $2 n$ are the same. So $\left|R^{k, x}-R^{k, x^{\prime}}\right|<\bar{\varepsilon} / 2$. But if $k$ is large enough, all but $\bar{\varepsilon} / 2$ of the $x$ 's have strings of length $2 n$ which are the same of the string for some $T_{B}^{Z} \bar{x}$ for a fixed $\bar{x}$. Thus, for this fixed $\bar{x},\left|R^{k, \bar{x}}-R^{k, x}\right|<\bar{\varepsilon}$ for all $x$ 's except a family of measure less than $\bar{\varepsilon} / 2$. This and the ergodic theorem give that the $R^{k, \bar{x}}$ name of most $y_{\alpha}$ agrees with the $R^{\beta, k}$ name of $P_{2} \phi\left(x, y_{\beta}\right)$ for most $x$. Thus $g_{\alpha}(k)=g_{\beta}(k)$, but $k$ is any sufficiently large integer and so $\bar{T}_{\alpha} \cong \bar{T}_{\beta}$.

\section{REFERENCES}

1. D. S. Ornstein, Bernoulli shifts with the same entropy are isomorphic, Advances in Math., 4 (1970), 337-352. 
2. D. S. Ornstein, Factors of a Bernoulli shifts are Bernoulli shifts, Advances in Math., 5 (1970), 349-364.

3. - Factors of a Bernoulli shift, Israel J. Math., 21 (1975), 145-153.

4. - Ergodic Theory, Randomness, and Dynamical Systems, Yale University Press, 1974.

5. D. S. Ornstein, and P. C. Shields, An uncountable family of K-automorphisms, Advances in Math., 10 (1973), 63-68.

6. D. S. Ornstein, and B. Weiss, Finitely determined implies very weak Bernoulli, Israel J. Math., 17. (1974), 94-104.

7. J.-P. Thouvenot, Quelques propriétés des systèmes dynamiques qui se décomposent en deux systèmes dont l'un est un schéma de Bernoulli, Israel J. Math., 21 (1975), 177-207.

Received May 17, 1976. Supported by NSF grant No. MCS 76-06005.

TeXas A \& M University

College Station, TX 77843 


\section{PACIFIC JOURNAL OF MATHEMATICS}

\section{EDITORS}

RICHARD ARENS (Managing Editor)

University of California

Los Angeles, California 90024

C. W. CURTIS

University of Oregon

Eugene, OR 97403

C. C. MOORE

University of California

Berkeley, CA 94720

\section{J. DugundJI}

Department of Mathematics University of Southern Californıa Los Angeles, California 90007

R. FinN AND J. Milgram Stanford University Stanford, California 94305

\section{ASSOCIATE EDITORS}

E. F. BECKENBACH

B. H. NEUMANN

F. WOLF

K. YOSHIDA

\section{SUPPORTING INSTITUTIONS}

UNIVERSITY OF BRITISH COLUMBIA CALIFORNIA INSTITUTE OF TECHNOLOGY UNIVERSITY OF CALIFORNIA MONTANA STATE UNIVERSITY UNIVERSITY OF NEVADA, RENO NEW MEXICO STATE UNIVERSITY OREGON STATE UNIVERSITY UNIVERSITY OF OREGON OSAKA UNIVERSITY
UNIVERSITY OF SOUTHERN CALIFORNIA STANFORD UNIVERSITY UNIVERSITY OF TOKYO UNIVERSITY OF UTAH WASHINGTON STATE UNIVERSITY UNIVERSITY OF WASHINGTON AMERICAN MATHEMATICAL SOCIETY NAVAL WEAPONS CENTER 


\section{Pacific Journal of Mathematics}

\section{Vol. 71, No. $1 \quad$ November, 1977}

Charalambos D. Aliprantis and Owen Sidney Burkinshaw, On universally complete Riesz spaces ............................. 1

Stephen Richard Bernfeld and Jagdish Chandra, Minimal and maximal solutions of nonlinear boundary value problems .................

John H. E. Cohn, The length of the period of the simple continued fraction of

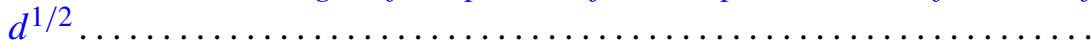

Earl Vern Dudley, Sidon sets associated with a closed subset of a compact abelian group .................................... 33

Larry Finkelstein, Finite groups with a standard component of type $J_{4} \ldots \ldots$

Louise Hay, Alfred Berry Manaster and Joseph Goeffrey Rosenstein, Concerning partial recursive similarity transformations of linearly ordered sets .......................................

Richard Michael Kane, On loop spaces without $p$ torsion. II ............

William A. Kirk and Rainald Schoneberg, Some results on

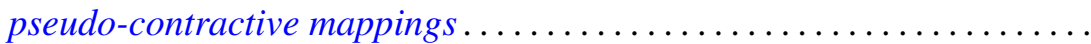

Philip A. Leonard and Kenneth S. Williams, The quadratic and quartic character of certain quadratic units. I. .

Lawrence Carlton Moore, A comparison of the relative uniform topology and the norm topology in a normed Riesz space .................

Mario Petrich, Maximal submonoids of the translational hull 119

Mark Bernard Ramras, Constructing new R-sequences . . .

Dave Riffelmacher, Multiplication alteration and related rigidity properties

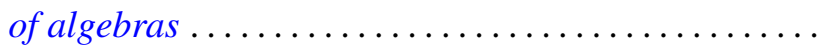

Jan Rosiński and Wojbor Woyczynski, Weakly orthogonally additive functionals, white noise integrals and linear Gaussian stochastic processes.

Ryōtarō Satō, Invariant measures for ergodic semigroups of operators

Peter John Slater and William Yslas Vélez, Permutations of the positive integers with restrictions on the sequence of differences...

Edith Twining Stevenson, Integral representations of algebraic cohomology classes on hypersurfaces ........................

Laif Swanson, Generators of factors of Bernoulli shifts . 